\title{
Numerical models for the elastic halfspace dynamic response due to road traffic load
}

\author{
Daniel Papán* and Zuazana Papánová \\ University of Žilina, Faculty of Civil Engineering, Department of Structural Mechanics and Applied \\ Mathematics, Univerzitná 8215/1, 010 26, Žilina, Slovakia
}

\begin{abstract}
Due to the information technology development the numerical modelling in the traffic structures engineering finding the application. Even in the traffic effect on the geological environment and soil. It this case two mechanical systems are interactive and acting to each other. This special type of interactive task is usually solves by using Finite Element Method (FEM). In both mechanical systems propagates the dynamic strength and strain effects. The Traffic Seismicity (TF) in the elastic half space is possible to solve in the time and the frequency domain. The paper contains theoretical approach of the two numerical simulations in the TS task. For this type of the FEM simulations the various numerical systems were used.
\end{abstract}

\section{Introduction}

The aim of this paper was to present some possible approaches to the numerical simulations of the dynamic tasks. These dynamic simulations are applied to solution of the passing of the road vehicles on the elastic half space model. The two types of different approaches to FEM modelling in this research field are presented in this paper.

The first way of the solution is application of the dynamic load in several points of the simplified hemisphere shaped FEM model. It means to solve a kinematic excitation in single point of 3D model. The dynamic load is applied as the measured movement of the real passing of the vehicle using in-situ test. These measurements are realized near to the source of the vibration. Analyses of this type can be called the experimental-theoretical combine analysis.

The second type of numerical modelling of this difficult problem is more sophisticated. In this case it is necessary to use two FEM systems. This way is only theoretical way. One mechanical system simulates the vehicle passing on the road and the results are using as a dynamic load to the second model. The second model simulates the response of the vehicle passing using the results of the first model. The second FEM model receives the response due to traffic seismicity effect. [1,2]

\footnotetext{
* Corresponding author: daniel.papan@fstav.uniza.sk
} 


\section{Numerical model of the half hemisphere homogeneous half space - model 1}

The first model of the elastic half space was modelled as hemisphere with diameter $r=50 \mathrm{~m}$ with stiff supports (Fig.1). The firs it was necessary to create the geometry in common computing AutoCAD 3D. Then the geometry was exported to the FEM system VisualFEA [3]. The depth of the modelled subsoil was according $Y=50 \mathrm{~m}$ and the length in horizontal direction were $X=100 \mathrm{~m}$, and in the perpendicular direction were $Z=100 \mathrm{~m}$, and it was defined in one layer. In this type of the model and sensitive set of the parameters the negative effects were eliminate (attenuation, reflections...). The dynamic load was applied in the centre of the circle upper surface in point $B I$ and in the distance $15 \mathrm{~m}$. The response of the simulation was compared with measured in points $B 3, B 4, B 5$ situated in between points $B 1$ and $B 2$. Material elastic properties were experimentally identified as: elastic modulus of the soil $E=4,5.10^{7} \mathrm{Nm}^{-2}$, unit mass $\rho=2000 \mathrm{~kg} \cdot \mathrm{m}^{-3}$ and the Poisson's constant $v=0,3$. As the finite elements used in this type of model the 4-points tetrahedrons were used.

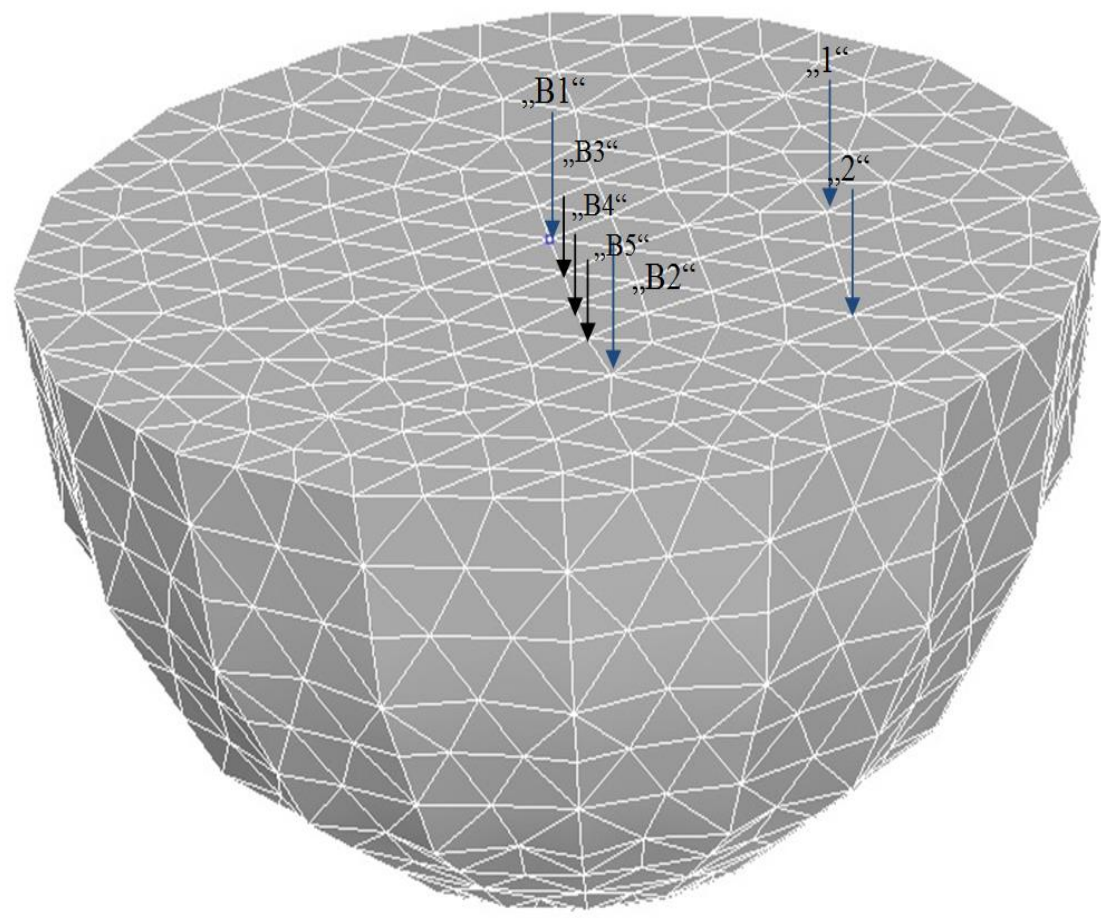

Fig. 1. The FE model of the hemisphere elastic half homogenous space.

The dynamic effect of the load was simulated on the model as application of the kinematic excitation. The measured time histories records were applied to B1 point on the model. Various types of the dynamic load were applied (mechanical impact, passing vehicle....). In the time domain calculation, the time histories were resulted and compared with measured. Using spectral analysis, the theoretical and experimental results was transformed to the frequency domain. Also the sensitive frequency bands were calculated as natural frequencies and the modes of the natural vibration. [4] 


\section{Two-layer model of elastic half space cuboid shape - model 2}

The second numerical model is created as two layered cuboid shaped elastic half space (Fig. 2). As a roadway layer the $0,48 \mathrm{~m}$ thickness of thin cuboid were modelled on the top on the soil model. The subsoil layer was modelled as a $10 \mathrm{~m}$ thick layer of the cuboid. The next layer $10 \mathrm{~m}$ thick models the stiff rock soil. The cuboid dimensions were: length $100 \mathrm{~m}$, width $25 \mathrm{~m}$ and the road width was $6 \mathrm{~m}$. This numerical model was also modelled in the same FEM system as in the capture 2. The stiff layer was supported stiff on the bottom of the cuboid. Material constants of each layer were set similar as in model 1 but they were layered.

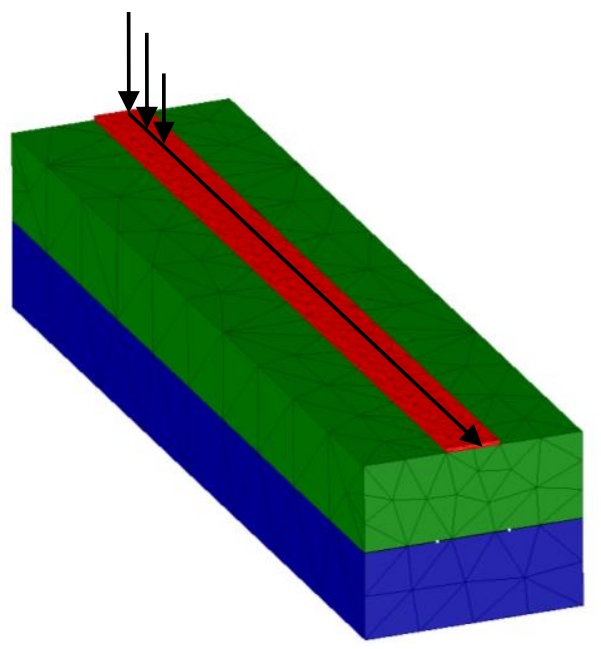

Fig. 2. The cuboid FEM model of the elastic half space

The dynamic load for this model was applied to 12 points on road surface line with the time shifted load function. This time domain load function was obtained from FEM system ADINA [5] as the result of the heavy vehicle moving simulation on surface with random unevenness. The load functions were time shifted depending on the distances of applied points. The time shift was set to simulate $60 \mathrm{~km} / \mathrm{h}$ vehicle speed. The load function was in form of vertical displacement in time. The dynamic load of model 2 is presented in Fig.3

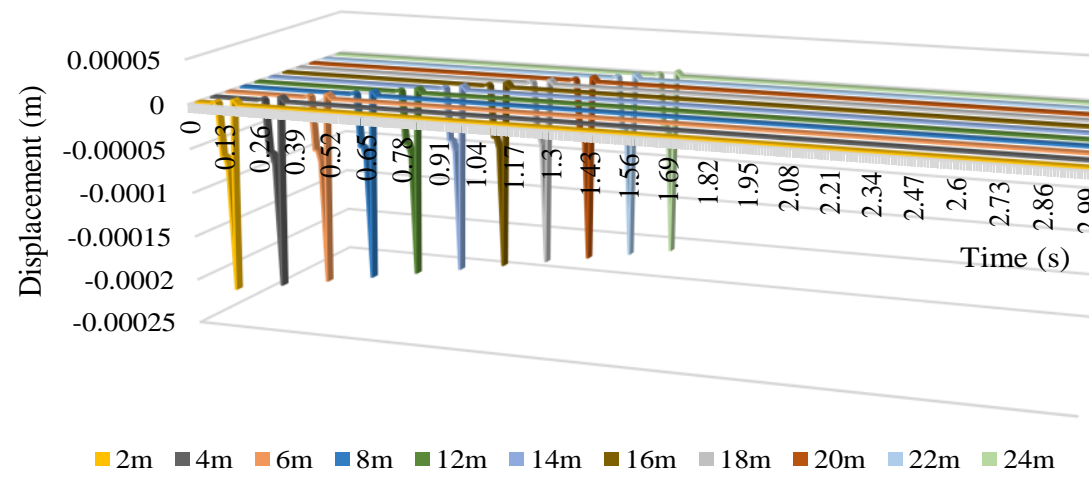

Fig. 3 The dynamic load of model 2 


\section{Comparison of the results of the numerical solutions}

The results of the time domain FEM simulation obtained from VisualFEA system in model 1 are showed as an example in Fig.4. This figure represents the modelled half space dynamic response due to applied measured load. It shows comparison numerical and experimental response in vibration velocity time history form.
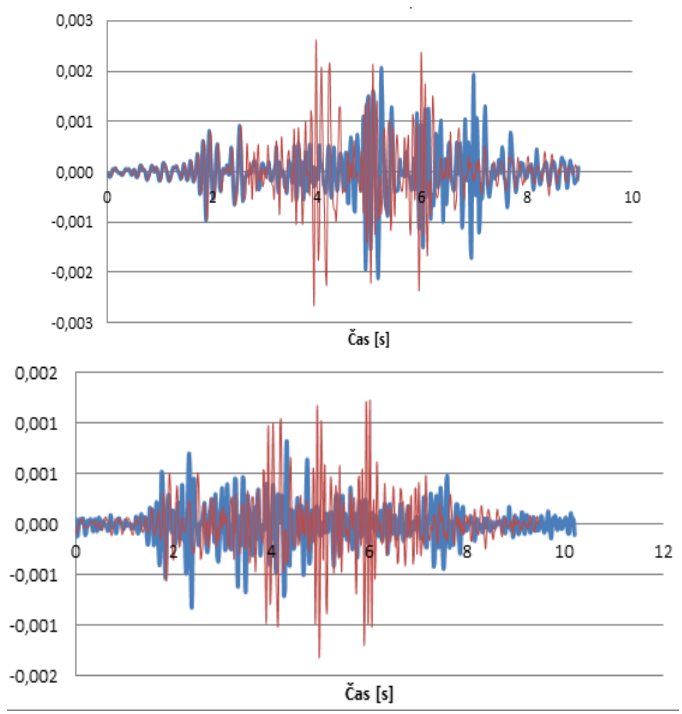

Fig. 4 Comparison of the results obtained from model 1 - vertical vibration velocities "point 1" (experimental red, numerical blue)

The spectral characteristics of the vertical vibration acceleration in several points of model 2 were observed. The results of experimental measurements due to moving Tatra 815 vehicle - averaged autospectral power densities are presented in Fig. 6. The peaks accelerations powers depending on the frequency are covered by dash dot curve.
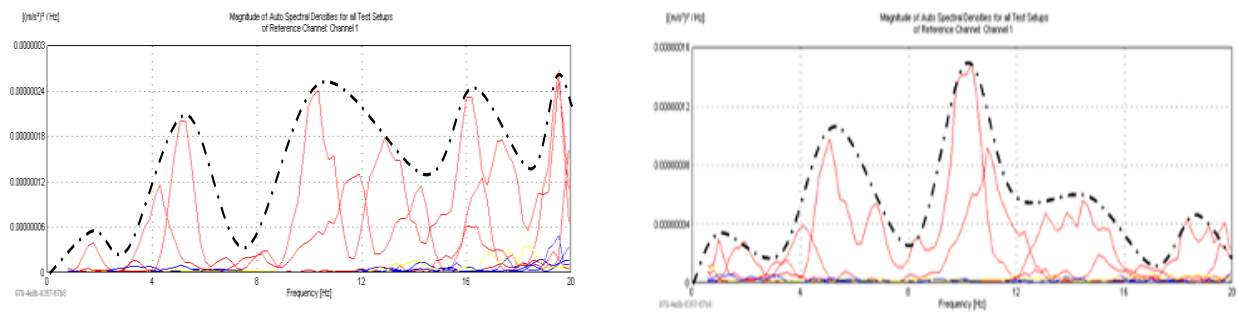

Fig. 5 Measured power spectral densities of the vertical acceleration due to Tatra heavy vehicle in $25 \mathrm{~m}$ distance to road

For better illustration of results interpretation the relevant vertical acceleration time histories in points with distance $25 \mathrm{~m}$ from loading line are presented in Fig.6. 

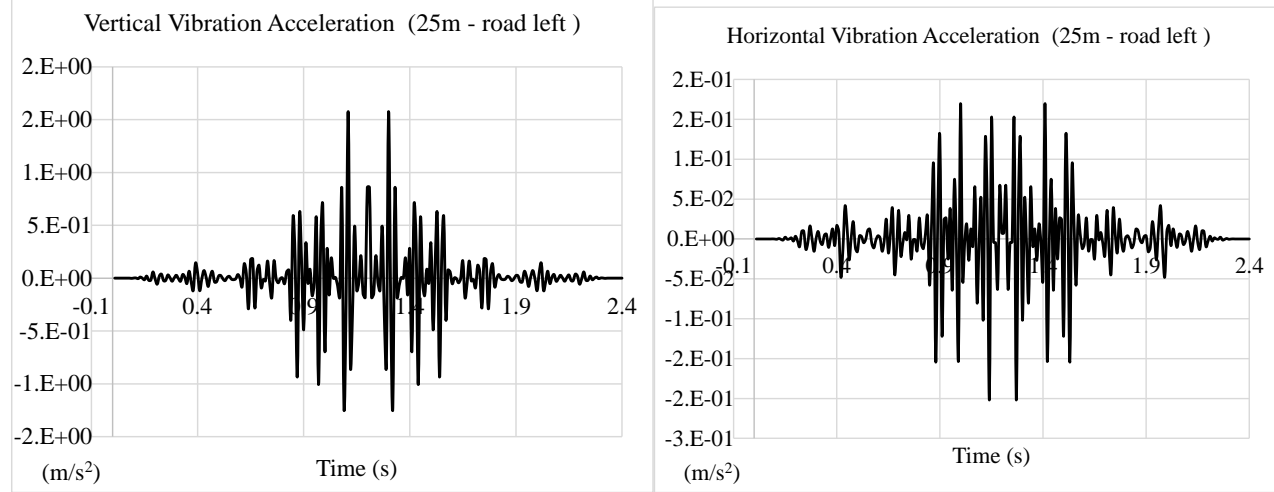

Fig. 6 Vertical acceleration time histories 25m distance to moving vehicle line FEM simulation results example (model 2)

The experimental measurements of the vibration accelerations in $25 \mathrm{~m}$ distance to road due to moving real Tatra vehicle was not compared. The comparison of the time domain results were not presented because the records were filtered. That is the reason to comparison in frequency domain for model 2. This comparison is presented in Fig. 7.
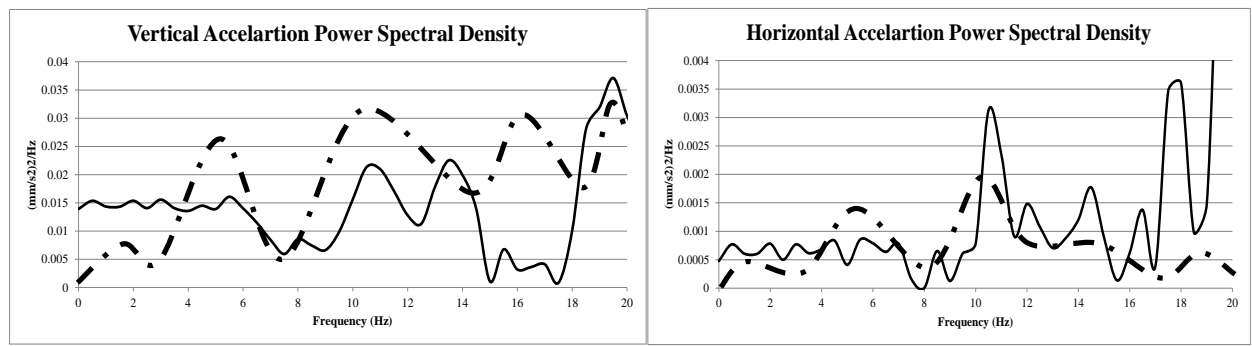

Fig. 7 Comparison of FEM and experimental results in frequency domain for model 2

\section{Conclusions}

Nowadays it is necessary to highlight the possibility of more difficult finite element modelling using three-dimensional elements and also this is utilised in structural dynamics. This advantage is more accessible on standard personal computers; desktops even in common hardware tools. Of course the accent on precision and theoretical approach to special problematics is needed. Also the relevance of the inputs to is type of task is important. This paper contains only small example to solution of numerical simulation of the traffic seismicity propagation in homogenous elastic half space model. There are two types of possible approach presented. Both types of FEM simulation are used in technical practice to some extent of precision. The result can be used for time extrapolation of traffic effects in cases of increasing of traffic intensity, increasing of road unevenness etc. There is some disproportionality in the result interpretation because of inhomogeneity of real geological environment and differences of traffic load. Despite a wide possibility of numerical methods using the experimental verification is more and more preferred.

This paper was created with the support of the OP Education for the project „Education quality support and research for the transport sector as the engine of the economy" (ITMS: 26110230076), which is co-financed by the European Social Fund. 


\section{References}

1. J.S. Bendat, A.G. Piersol, Engineering Applications of Correlation and Spectral Analysis (Wiley, New York, 1980)

2. J. Benčat, Predictions and analysis of dynamic effects of traffic on structures and environment (Grand Task 1/0169/12, VEGA - UNIZA, 2012-2014)

3. VISUAL FEA Theoretical manual, http://visualfea.com/onlinemanual.htm

4. D. Papán, Z. Papánová, Simulation of the vibration propagation in elastic halfspace due to traffic seismicity in time domain ( Physical factors of environment, Vol.4, 2014)

5. D. Papán, V. Valášková, Assessment of the roadway dynamic response due to the Tatra 815 lorry excitation and experimental verification, (Communication - scientific letters, Vol.4, 2016) 